MODELING, IDENTIFICATION AND CONTROL, 1988, vOL. 9, NO. 2, 59-68

doi:10.4173/mic.1988.21

\title{
Adaptive control of a manipulator when the mass of the load is unknown
}

\author{
OLAV EGELAND†
}

Keywords: Robots, adaptive control, parameter estimation.

\begin{abstract}
An adaptive controller for a robotic manipulator is presented. In the control system, the computed torque technique is used to linearize and decouple the dynamic model. The computed torque technique requires that the complete dynamic model is known. Normally, this model is known except perhaps for a few parameters, e.g. load inertia and centre of gravity and friction co-efficients. In this paper, the unknown parameters, which all represent physical quantities, are estimated using a recursive prediction error method. The most recent parameter estimate is then used in the computed torque technique. The control system is applied to the positioning part of an industrial robot in a simulation experiment where the unknown parameter is the mass of the load. The controller performed well, and the mass of the load can even be estimated when the manipulator is accelerating and there is a large control deviation.
\end{abstract}

\section{Introduction}

The state space equations of motion of an $n$-link robotic manipulator are nonlinear and contain a large number of terms. However, by using the computed torque technique (Bejczy, 1974), a linear time-invariant state space model consisting of $n$ decoupled double integrators is obtained. The controller design for these decoupled double integrators is straightforward.

In order to apply the computed torque technique, the dynamic model of the manipulator must be known. This model can be found from Newton-Euler's equations of motion (Luh, Walker and Paul, 1980). The parameters of this model are the link and actuator inertias, the Denavit-Hartenberg parameters, friction parameters, and the centre of gravity and the inertia of the load. The link and actuator inertias and the Denavit-Hartenberg parameters are constant and can be found before the manipulator is installed. When this is not the case, these parameters can be found using parameter identification techniques before the manipulator is used in production. However, friction and load parameters may be time-varying and this may cause problems in the implementation of the computed torque technique.

The mass of the load can be found with a wrist force sensor (Paul, 1981). However, most industrial robots are not equipped with this type of sensor, and this solution has the drawback that the load must either be at rest or be moved at a constant speed while the mass of the load is measured. The mass of the load may also be found from the joint torques, but in this case also, the load must not be accelerated while the torques are measured.

Adaptive control of manipulators has been proposed. Koivo and Guo (1983) use a minimum variance controller based on a linear ARMAX model and Landau

Received March 9, 1987.

This paper was presented at the IFAC Int. Symposium on Theory of Robots, December 3-5, 1986, Vienna, Austria and is reprinted with permission of IFAC.

† The Norwegian Institute of Technology, Division of Engineering Cybernetics, N-7034 Trondheim-NTH, Norway. 
(1985) uses model reference adaptive control. In these techniques, the dynamic model of the manipulator is assumed to be unknown, and quantities which are functions of joint positions and velocities are assumed to be slowly varying parameters.

Craig, Hsu and Sastry (1986) present an adaptive controller which takes full advantage of any known parameter, while estimating remaining unknown parameters. The computed torque technique is used, and the unknown parameters are estimated using a model reference adaptive control technique.

In this paper, an adaptive controller is presented which is based upon the same ideas as the one proposed by Craig et al. (1986). The main difference between the two adaptive controllers is the parameter estimation algorithm, which in the present paper is a recursive prediction error method. This means that here the parameter estimates are updated from the prediction error, while in Craig et al. (1986), the parameter estimates are updated from the control deviation. In periods where the control deviation is large, such as when there is a step in the position reference, updating from the prediction error will be better than updating from the control deviation. This situation may occur when an unknown load is to be lifted.

When the mass of the load is to be found, the proposed adaptive algorithm has the advantage that the manipulator need not move in a specified way as when the mass is to be found from a wrist force sensor or the joint torques.

The paper is organized as follows: First the computed torque technique is reviewed. Then the parameter estimation algorithm is developed, and finally the performance of the control system is demonstrated in a simulation experiment.

\section{The computed torque technique}

The equations of motion for a general $n$-link manipulator can be found from Newton-Euler's equation (Luh et al. 1980), and is written

$$
M(\boldsymbol{q} ; \boldsymbol{\theta}) \ddot{\boldsymbol{q}}=\boldsymbol{n}(\boldsymbol{q}, \dot{\boldsymbol{q}} ; \boldsymbol{\theta})+\boldsymbol{\tau}
$$

where $q$ is the vector of joint displacements, $M(q ; \theta)$ is the inertia matrix, $\boldsymbol{n}(\boldsymbol{q}, \dot{\boldsymbol{q}} ; \boldsymbol{\theta})$ is a vector defining friction, gravity, Coriolis and centrifugal terms, and $\tau$ is the vector of input generalized forces in the joints. $\boldsymbol{\theta}$ is a parameter vector.

First, we assume that the parameter vector $\theta$ is known. In the computed torque technique, the input generalized forces are chosen as

$$
\boldsymbol{\tau}=M(\boldsymbol{q}) ; \boldsymbol{\theta}) \boldsymbol{u}-\boldsymbol{n}(\boldsymbol{q}, \dot{\boldsymbol{q}} ; \boldsymbol{\theta})
$$

where $\boldsymbol{u}$ is the transformed control vector.

By inserting (2) into (1), we get the linear time-invariant system

$$
\ddot{\boldsymbol{q}}=\boldsymbol{u}
$$

or in state space form

$$
\begin{gathered}
\dot{x}_{1}=x_{2} \\
\dot{x}_{2}=u
\end{gathered}
$$

where $\boldsymbol{x}_{1}=\boldsymbol{q}$ and $\boldsymbol{x}_{2}=\dot{\boldsymbol{q}}$.

The problem of finding a controller for the system described by (1) is now reduced to the much simpler problem of finding a controller for each of the $\boldsymbol{n}$ decoupled double integrators in (4). 
In this paper we assume that $\boldsymbol{u}$ is generated by state feedback:

$$
u=G_{1} \Delta x_{1}+G_{2} \Delta x_{2}
$$

where $\Delta x_{1}$ and $\Delta x_{2}$ are the control deviations $\Delta x_{1}=x_{1}-x_{1, \text { ref }}$ and $\Delta x_{2}=$ $x_{2}-x_{2, \text { ref }}$ where $x_{1}$, ref and $x_{2, \text { ref }}$ are the position and velocity references. $G_{1}$ and $G_{2}$ are constant diagonal gain matrices. The diagonal terms of $G_{1}$ and $G_{2}$ can be chosen from classical servomechanism theory, pole placement techniques or linear quadratic optimal control.

We now consider the case where the parameter vector $\theta$ is unknown. In the computed torque technique, an estimate $\hat{\boldsymbol{\theta}}$ of $\boldsymbol{\theta}$ must be used. The input generalized forces are then chosen as

$$
\boldsymbol{\tau}=M(\boldsymbol{q} ; \hat{\boldsymbol{\theta}}) \boldsymbol{u}+\boldsymbol{n}(\boldsymbol{q}, \dot{\boldsymbol{q}} ; \hat{\boldsymbol{\theta}})
$$

For brevity, we introduce the notation $M=M(\boldsymbol{q} ; \boldsymbol{\theta}), \boldsymbol{n}=\boldsymbol{n}(\boldsymbol{q}, \dot{\boldsymbol{q}} ; \boldsymbol{\theta}), \hat{M}=M(\boldsymbol{q} ; \hat{\boldsymbol{\theta}})$ and $\hat{\boldsymbol{n}}=\boldsymbol{n}(\boldsymbol{q}, \dot{\boldsymbol{q}} ; \hat{\boldsymbol{\theta}})$. Because the estimated inertia matrix $\hat{M}$ is equal to the inertia matrix of the manipulator when $\boldsymbol{\theta}=\hat{\boldsymbol{\theta}}$, it follows that $\hat{M}$ is always symmetric and positive definite.

By inserting (6) into (1), we get the state space model

$$
\begin{gathered}
\dot{\boldsymbol{x}}_{1}=\boldsymbol{x}_{2} \\
\dot{\boldsymbol{x}}_{2}=\boldsymbol{u}+M^{-1}[\boldsymbol{n}-\hat{\boldsymbol{n}}+(\hat{M}-M) \boldsymbol{u}]
\end{gathered}
$$

We see that when our parameter estimate $\hat{\boldsymbol{\theta}}$ is not equal to the correct value $\boldsymbol{\theta}$, the computed torque technique fails to linearize and decouple the system.

\section{Parameter estimation}

A commonly used parameter estimation algorithm in adaptive controllers is the recursive prediction error method (Ljung and Söderström, 1983). Here the extended Kalman filter formulation of the algorithm is used:

$$
\begin{gathered}
\hat{\boldsymbol{\theta}}(k+1)=\hat{\boldsymbol{\theta}}(k)+K(k+1) \varepsilon(k+1) \\
K(k+1)=P(k) \Psi(k+1)\left[\Psi^{T}(k+1) P(k) \Psi(k+1)+W\right]^{-1} \\
P(k+1)=\left[I-K(k+1) \Psi^{T}(k+1)\right] P(k)+V
\end{gathered}
$$

where $\hat{\boldsymbol{\theta}}(k)$ is an estimate at time $k$ of the parameter vector $\boldsymbol{\theta}, K(k)$ is the updating gain, $\boldsymbol{\varepsilon}(k)=\boldsymbol{y}(k)-\overline{\boldsymbol{y}}(k)$ is the prediction error, where $\boldsymbol{y}(k)$ is the actual process measurement, and $\bar{y}(k)$ is a measurement prediction generated by a process model. $P(k)$ is an estimate of $\operatorname{cov}(\hat{\boldsymbol{\theta}}(k))$, and $\Psi(k)=-\partial \boldsymbol{\varepsilon}(k) / \partial \hat{\boldsymbol{\theta}}(k)$ is the sensitivity matrix.

The sensitivities are given in terms of old values of control inputs, $\boldsymbol{u}(k)$, and measurements, $\boldsymbol{y}(k)$, when an ARMA model and a least squares method is used. In our case, $\Psi(k)$ is computed by solving a set of sensitivity equations (Sælid, Jenssen and Balchen, 1983).

In order to track a time varying $\boldsymbol{\theta}$, the parameter vector is modeled as a Wiener process

$$
\boldsymbol{\theta}(k+1)=\boldsymbol{\theta}(k)+\boldsymbol{v}(k)
$$

where $\boldsymbol{v}(k)$ is a white noise process with covariance $\operatorname{cov}(\boldsymbol{v}(k))=V$.

The measurement is modeled as

$$
\boldsymbol{y}(k)=D_{1} x_{1}(k)+D_{2} x_{2}(k)+w(k)
$$


where $\boldsymbol{w}(k)$ is a white noise process with covariance $\operatorname{cov}(\boldsymbol{w}(k))=W$.

We first consider the case where only joint displacements are measured, which means that

$$
y=x_{1}+w
$$

The measurement prediction is generated by a state estimator.

We discretize the model (7) using the Euler method with sampling interval $T$ :

$$
\begin{aligned}
x_{1}(k+1)= & x_{1}(k)+T x_{2}(k) \\
x_{2}(k+1)= & x_{2}(k)+T u(k) \\
& +T M^{-1}[n-\hat{n}+(\hat{M}-M) u(k)]
\end{aligned}
$$

The state estimator is

$$
\begin{aligned}
\overline{\boldsymbol{x}}_{1}(k+1) & =\hat{\boldsymbol{x}}_{1}(k)+T \hat{\boldsymbol{x}}_{2}(k) \\
\overline{\boldsymbol{x}}_{2}(k+1) & =\hat{\boldsymbol{x}}_{2}(k)+T u(k) \\
\overline{\boldsymbol{y}}(k) & =\overline{\boldsymbol{x}}_{1}(k) \\
\varepsilon(k) & =\boldsymbol{y}(k)-\overline{\boldsymbol{y}}(k) \\
\hat{\boldsymbol{x}}_{1}(k) & =\overline{\boldsymbol{x}}_{1}(k)+K_{1} \varepsilon(k) \\
\hat{\boldsymbol{x}}_{2}(k) & =\overline{\boldsymbol{x}}_{2}(k)+K_{2} \varepsilon(k)
\end{aligned}
$$

where $\overline{\boldsymbol{x}}_{1}$ and $\overline{\boldsymbol{x}}_{2}$ are the a priori estimates of $\boldsymbol{x}_{1}$ and $\boldsymbol{x}_{2}$, while $\hat{\boldsymbol{x}}_{1}$ and $\hat{\boldsymbol{x}}_{2}$ are the $a$ posteriori estimates. $K_{1}$ and $K_{2}$ are constant diagonal matrices.

As only position is measured, state feedback as given by (5) is not possible. Instead, feedback is taken from the a posteriori estimate of the state vector

$$
\begin{aligned}
u(k)= & G_{1}^{d}\left(\hat{x}_{1}(k)-x_{1, \text { ref }}(k)\right) \\
& +G_{2}^{d}\left(\hat{x}_{2}(k)-x_{2, \text { ref }}(k)\right)
\end{aligned}
$$

where $G_{1}^{d}$ and $G_{2}^{d}$ are constant diagonal gain matrices.

The sensitivity matrix $\Psi$ is computed by differentiating the equations for $\tilde{\boldsymbol{x}}_{1}=$ $\overline{\boldsymbol{x}}_{1}-\boldsymbol{x}_{1}$ and $\tilde{\boldsymbol{x}}_{2}=\overline{\boldsymbol{x}}_{2}-\boldsymbol{x}_{2}$ with respect to $\hat{\boldsymbol{\theta}}$. We obtain from eqns. (13)-(17)

$$
\begin{aligned}
Z_{1}(k+1)= & \left(I-K_{1}-T K_{2}\right) Z_{1}(k)+T Z_{2}(k) \\
Z_{2}(k+1)= & -K_{2} Z_{1}(k)+Z_{2}(k) \\
& -T M^{-1}\left[-\frac{\partial \hat{n}}{\partial \hat{\theta}}+\frac{\partial \hat{M}}{\partial \hat{\theta}} \boldsymbol{u}(k)\right] \\
\Psi(k)= & Z_{1}(k)
\end{aligned}
$$

where $Z_{1}=\partial \tilde{\boldsymbol{x}}_{1} / \partial \hat{\boldsymbol{\theta}}$ and $Z_{2}=\partial \tilde{\boldsymbol{x}}_{2} / \partial \hat{\boldsymbol{\theta}}$.

The total parameter estimation algorithm consists of (8), (9), and (10), where the parameter estimates are computed, the state estimator given by (14), (15), (16), and (17), and the sensitivity equations (19) and (20). The computational requirements of the algorithm are very modest except for the last term in (19b), and to some extent (9) and (10). The adaptive control system is shown in Fig. 1.

We now consider the case where the joint velocities are also measured. We also assume that the measurement noise is negligible. As a consequence of this, we let the 


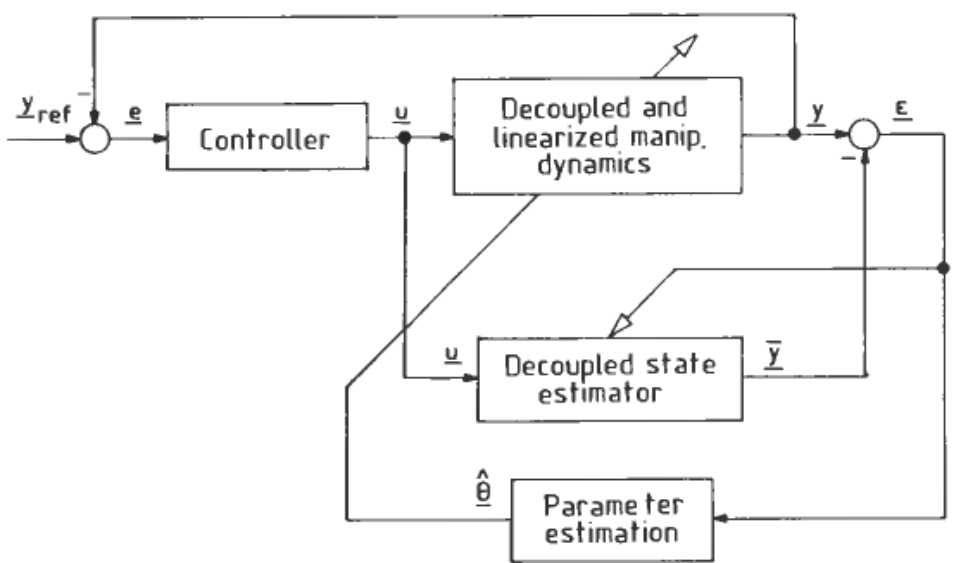

Figure 1. Adaptive controller.

a posteriori estimates be given by

$$
\begin{aligned}
& \hat{x}_{1}(k)=x_{1}(k) \\
& \hat{x}_{2}(k)=x_{2}(k)
\end{aligned}
$$

We now get from (13a), (14a), and (21a)

$$
\tilde{\boldsymbol{x}}_{\mathbf{1}}(k) \equiv \mathbf{0}
$$

which means that the position prediction error $\varepsilon_{1}$ is not sensitive to variations in $\hat{\theta}$. This means that the parameter estimates are only updated from the velocity prediction error $\varepsilon_{2}$. The sensitivity equations are

$$
\begin{aligned}
Z_{1}(k) & \equiv 0 \\
Z_{2}(k+1) & =T M^{-1}\left[-\frac{\partial \hat{n}}{\partial \hat{\theta}}+\frac{\partial \hat{M}}{\partial \hat{\boldsymbol{\theta}}} \boldsymbol{u}(k)\right] \\
\Psi(k) & =Z_{2}(k)
\end{aligned}
$$

The parameter estimation algorithm now consists of (8), (9), and (10), the velocity estimator is given by (14b) and (21b), and the sensitivity equations (23b) and (24).

As stated above, the computation of the term

$$
M^{-1}\left[-\frac{\partial \hat{\boldsymbol{n}}}{\partial \hat{\boldsymbol{\theta}}}+\frac{\partial \hat{M}}{\partial \hat{\boldsymbol{\theta}}} \boldsymbol{u}(k)\right]
$$

may cause problems in a real time system. For one parameter, the computational requirements will be comparable to the computed torque technique. It is clear that with current state-of-the-art micro-processors, only a small number of parameters may be estimated for a 6-link manipulator.

In the computed torque technique, the input generalized forces $\tau$ in (2) can be computed using the recursive Newton-Euler scheme (Luh et al. 1980). However, in this parameter estimation algorithm, an analytical model is required to compute the 


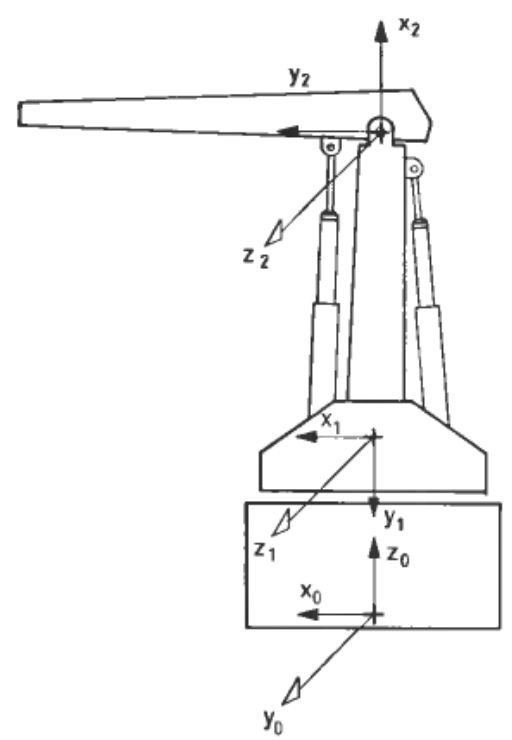

Figure 2. Positioning part of an industrial manipulator.

inertia matrix $M$ and the partial derivatives $\partial \hat{\boldsymbol{n}} / \partial \hat{\boldsymbol{\theta}}$ and $\partial \hat{M} / \partial \hat{\boldsymbol{\theta}}$. The generation of analytical dynamic models for manipulators by hand is a very lengthy process except for manipulators with three joints or less.

The need for an analytical model is a weak point. However, this problem can be solved by using an analytical model generator (e.g. Burdick, 1986) and symbolic computation to find the partial derivatives of $\hat{\boldsymbol{n}}$ and $\hat{M}$.

In an adaptive control system for an industrial manipulator, there will be few unknown parameters. In many cases the only unknown parameter will be the mass of an unknown load. In this case, it should be possible to run the proposed parameter estimation algorithm in real time.

Parameter estimation algorithms are often derived under the assumption that there is no feedback. This is because in a closed loop, there may be problems with parameter identifiability. However, in the proposed adaptive controller, the parameter estimation algorithm is applied to the system consisting of the process with controller. As a result of this, there are no problems with closed loop identification.

Also, because the parameter estimation algorithm is applied to the system after the computed torque technique has decoupled and linearized the system, the resulting state estimator becomes very simple with very modest computational requirements.

\section{Simulation}

In a simulation experiment, the proposed algorithm was applied to the positioning part of an industrial robot with rotational joints, see Fig. 2 . The dynamic model of the manipulator is

$$
M\left(\boldsymbol{q} ; m_{L}\right) \ddot{\boldsymbol{q}}=\boldsymbol{n}\left(\boldsymbol{q}, \dot{\boldsymbol{q}} ; m_{L}\right)+\tau
$$


where

$$
\begin{aligned}
M & =\left[\begin{array}{ccc}
J_{1} & 0 & 0 \\
0 & J_{22}+2 J_{23} C_{3}+J_{33} & J_{23} C_{3}+J_{33} \\
0 & J_{23} C_{3}+J_{33} & J_{33}
\end{array}\right] \\
J_{1} & =J_{11}+J_{22} C_{2}^{2}+J_{33} C_{2+3}^{2}+J_{23} C_{2} C_{2+3} \\
n & =\left[n_{1}, n_{2}, n_{3}\right]^{T}
\end{aligned}
$$

where

$$
\begin{aligned}
n_{1}= & 2\left[J_{23} S_{2} C_{2+3}+J_{22} S_{2} C_{2}\right] \dot{q}_{1} \dot{q}_{2} \\
& +2\left[J_{23} S_{2+3} C_{2}+J_{33} S_{2+3} C_{2+3}\right] \dot{q}_{1}\left(\dot{q}_{2}+\dot{q}_{3}\right) \\
n_{2}= & -\left[J_{23}\left(C_{2+3} S_{2}+C_{2} S_{2+3}\right)+J_{22} C_{2} S_{2}\right. \\
& \left.+J_{33} C_{2+3} S_{2+3}\right] \dot{q}_{1}^{2}+J_{23} S_{3} \dot{q}_{3}^{2}+M_{2} C_{2} \\
& +2 M_{3} C_{2+3} \\
n_{3}= & -\left[J_{23} C_{2} S_{2+3}+J_{33} C_{2+3} S_{2+3}\right] \dot{q}_{1}^{2} \\
& -J_{23} S_{3} \dot{q}_{2}^{2}+M_{3} C_{2+3}
\end{aligned}
$$

Here $C_{2}=\cos q_{2}, C_{2+3}=\cos \left(q_{2}+q_{3}\right), C_{3}=\cos q_{3}, S_{2}=\sin q_{2}, S_{2+3}=$ $\sin \left(q_{2}+q_{3}\right)$ and $S_{3}=\sin q_{3} . J_{i j}$ are the appropriate moments of inertia and $M_{2}$ and $M_{3}$ are gravitational coefficients.

The computed torque was used. The feedback gain was chosen by pole placement with the desired poles being $\lambda_{1,2}=-22 \pm j 22$. This corresponds to

$$
G_{1}=-1000 I_{3}
$$

and

$$
G_{2}=-45 I_{3}
$$

in the continuous case, where $I_{3}$ is the three-dimensional identity matrix.

The unknown parameter was the mass, $m_{L}$, of a load which was a point mass located at the tip of link 3. In the dynamic model, the unknown parameter $m_{L}$ enters the parameters $J_{22}, J_{33}, J_{23}, M_{2}$, and $M_{3}$ linearly. The derivation of the sensitivity equations is straightforward, and the resulting equations have a structure which resembles the dynamic model.

To get an indication of the computational requirements, the adaptive control algorithm was run at our robot control system which has a Motorola 68020/68881 microprocessor. The execution time was about $2.5 \mathrm{~ms}$ when three joints were controlled and one parameter was unknown. With minor simplifications it will be possible to control six joints with one unknown parameter with a sampling interval of $10 \mathrm{~ms}$ on the same hardware.

The adaptive controller was first simulated with position measurements only. The updating gains in the state estimator were chosen as

$$
\begin{aligned}
& K_{1}=I_{3} \\
& K_{2}=0.2 T^{-1} I_{3}
\end{aligned}
$$




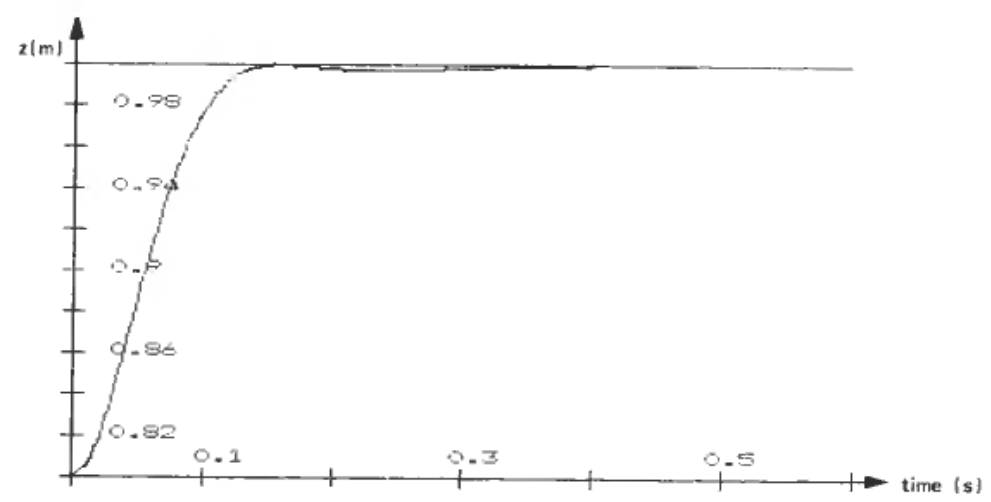

Figure 3. Step response in $z_{0}$ direction with only position measurements.

where $T$ is the sampling interval. This gives a state estimator with a high bandwidth.

The initial covariance of the parameter estimate was set to $P(0)=1$, and the noise covariance in the Wiener process (11) was chosen as $V=0 \cdot 01 I_{3}$. Measurement noise covariance in the position measurement was $W=1 \times 10^{-6} I_{3}$. The initial position of the manipulator was as shown in Fig. 3 where $q_{2}=-90^{\circ}$ and $q_{3}=90^{\circ}$. The $z_{0}$ position of the tip of link three was $0.8 \mathrm{~m}$. The reference in the $z_{0}$ direction was given a step change from $0.8 \mathrm{~m}$ to $1.0 \mathrm{~m}$ at the time $t=0$. The unknown mass, $m_{L}$, of the load was $2 \mathrm{~kg}$. The initial estimate of the mass was $0 \mathrm{~kg}$.

The step response in the $z_{0}$ direction of the manipulator with the adaptive control system is shown in Fig. 3. The parameter estimate $\hat{m}_{L}$, is shown in Fig. 4. We see that the parameter estimate converged fast even though the control deviation was large initially. The step response in the $z_{0}$ direction was fast considering the initial error in the parameter estimate.

The manipulator was then simulated with an adaptive control system with position and velocity measurements. The controller and noise parameters were chosen as above except for the measurement noise. The velocity measurement noise covariance was set to $W=0.01 I_{3}$ in the parameter estimation algorithm.

In this case, the step response in the $z_{0}$ direction was as shown in Fig. 5. The parameter estimate, $\hat{m}_{L}$, is shown in Fig. 6 . The results were somewhat better than in the case where only the position was measured.

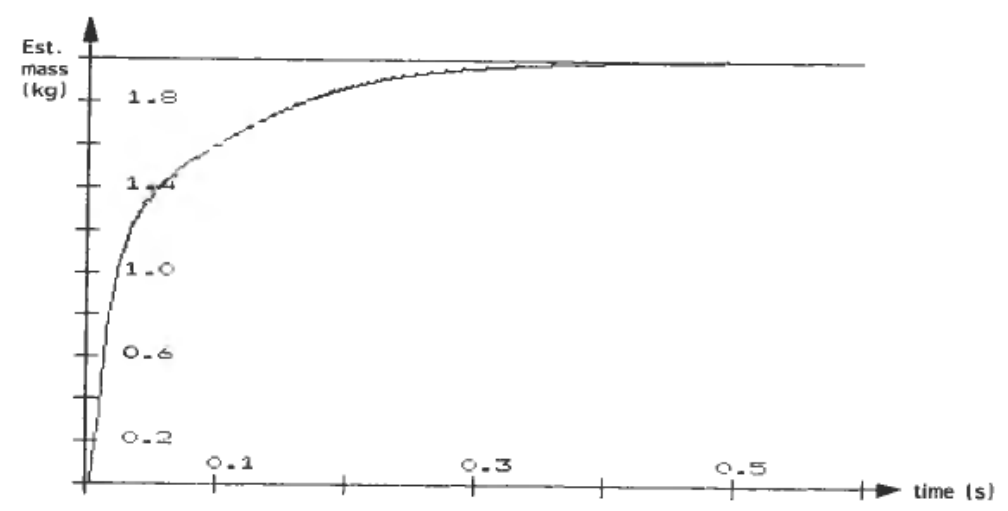

Figure 4. Estimated mass with only position measurements. 


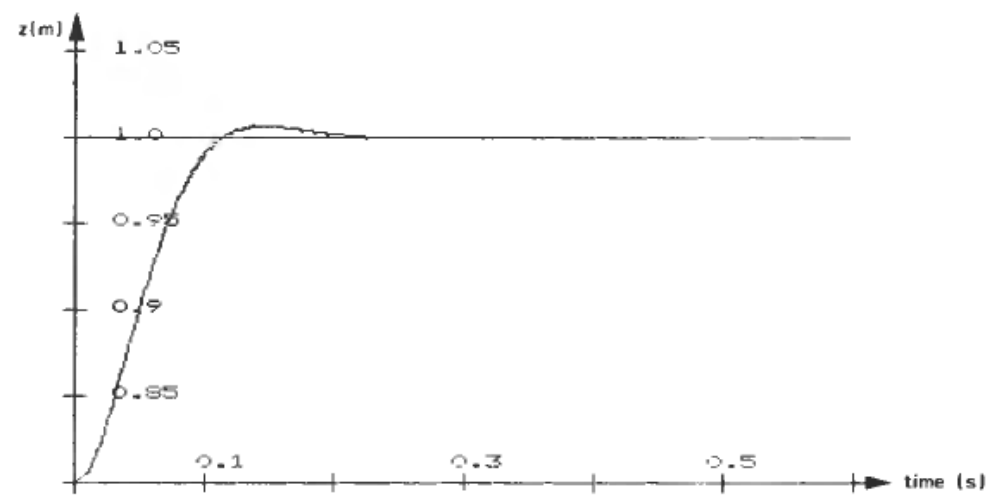

Figure 5. Step response in $z_{0}$ direction with position and velocity measurements.

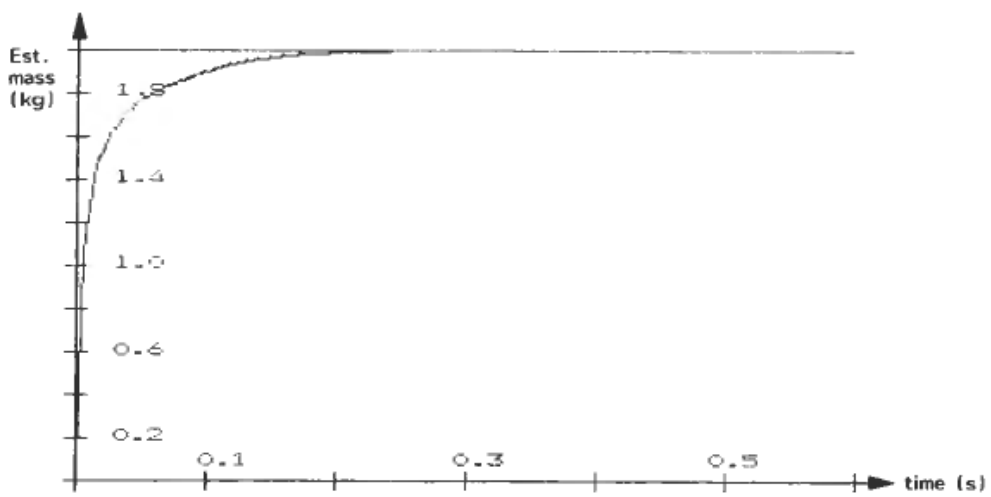

Figure 6. Estimated mass with position and velocity measurements.

\section{Conclusion}

An adaptive control system for a robotic manipulator has been presented. The control system uses the computed torque technique, and full advantage is taken of the known part of the dynamic model. The unknown parameters in the dynamic model are found from a recursive prediction error parameter estimation algorithm.

In the case of one unknown parameter, the computational requirements of the parameter estimation algorithm are comparable to those of the computed torque technique.

As the parameter estimates are updated from the prediction error, the parameter estimates converge even if the control deviations are large or if the manipulator is accelerated.

The adaptive control system performs very well in a simulation experiment where the mass of the load is the unknown parameter.

\section{ACKNOWLEDGEMENT}

This work has been sponsored by the Royal Norwegian Council for Scientific and Industrial Research through the Robotic research program ED331.12510. 


\section{REFERENCES}

BEJCZY, A. K. (1974). Robot arm dynamics and control. JPL TM 33-669.

BuRDICK, J. W. (1986). An algorithm for generation of efficient manipulator dynamic equations. Proc. 1986 Int. Conf. on Robotics and Automation, San Francisco, Calif. April 7-10, 1986.

Craig, J. J., Hsu, P. and Sastry, S. S. (1986). Adaptive control of mechanical manipulators. Proc. 1986 Int. Conf. on Robotics and Automation, San Francisco, Calif. April 7-10, 1986.

Korvo, A. J. and Guo, T.-H. (1983). Adaptive linear controller for robotic manipulators. IEEE Trans. Autom. Control, 28, 162-171.

LANDAU, I. D. (1985). Adaptive control techniques for robotic manipulators-the status of the art. Proc. IF AC Symp. on Robot Control, Barcelona, Nov. 6-8, 1985.

LJUnG, L. and SöDERSTRÖM, T. (1983). Theory and Practice of Recursive Identification. (The MIT Press; Cambridge, Massachusetts).

LuH, J. Y. S., Walker, M. W. and PaUl, R. P. C. (1980). On-line computational scheme for mechanical manipulators. J. Dynamic Systems, Measurement and Control, 102, 69-76.

PAUL, R. P. (1981). Robot manipulators: Mathematics, Programming and Control. (The MIT Press; Cambridge, Massachusetts).

Saelid, S., Jenssen, N. A. and Balchen, J. G. (1983). Design and analysis of a dynamic positioning system based on Kalman filtering and optimal control. IEEE Trans. Autom. Control, 28, 331-339. 